\title{
Triterpenes and other Metabolites from Tibouchina urvilleana
}

\section{Ana-Lidia Pérez-Castorena}

Instituto de Química, Universidad Nacional Autónoma de México, Circuito Exterior, Ciudad Universitaria, Coyoacán 04510, D. F., México. alperezc@unam.mx

Received August 20 $0^{\text {th }}, 2013$; Accepted April 3 ${ }^{\text {rd }}, 2014$

\begin{abstract}
The chemical study of the leaves and stems of Tibouchina urvilleana afforded triterpenes of different types: four oleanane, four ursane, one glutinane, and one taraxerane. Also, two flavonoids and two sterols were isolated. Additionally, the toxicity and topical antiinflammation activity of the extracts were tested.

Key Words: Tibouchina urvilleana, Melastomataceae, triterpenes, flavonoids, sterols.
\end{abstract}

\section{Introduction}

The genus Tibouchina Aubl. (Melastomataceae) comprises about 240 species distributed from southern Mexico to northern Argentina [1]. A characteristic of their flowers is the beautiful dark purple color; therefore, several species are currently cultivated as ornamental plants. Also, some species of Tibouchina are used in the popular medicine, as T. grandifolia whose tea from leaves is utilized to enhance wound healing in Brazil [2]. Although Tibouchina is a large genus only eight species have been investigated chemically. The results obtained so far point to phenolic compounds as substances characteristic of this genus. Flavonol glycosides as quercetin 3-O-rhamnopyranoside and quercetin 3-O- $\beta$-D-glucopyranoside have been isolated from the leaves of T. ciliaris [3], T. grandifolia [2], and $T$. semidecandra [4a]. Hydrolysable tannin oligomers, mainly ellegatannins known as nobotanins, were obtained from the stem barks of T. multiflora [5] and T. semidecandra [4b]. Also, tannins were detected on T. pulchra [6]. Additionally and in order to obtain compounds useful as natural food pigments, anthocyanins, the flowers of $T$. grandiflora [7], T. granulose [8a,b], $T$. semidecandra [4c], and T. urvilleana [9] were analyzed, obtaining malvidin and peonidin derivatives. Since only the chemical constituents of the flowers of $T$. urvilleana have been reported, the present work details the main secondary metabolites from the leaves and stems of this species, and additionally, the study contributes to the knowledge of the genus chemistry.

\section{Results and Discussion}

The leaves and stems of $T$. urvilleana were extracted successively with hexane, EtOAc, and $\mathrm{MeOH}$. Purification of the hexane extract permitted the isolation of glutinol (1) [10], taraxerol (2) [11], a mixture of $\alpha$ - and $\beta$-amyrins $(3,4)[11 \mathrm{~b}, 12], \beta$-sitosterol, and ursolic (5) [13] and oleanolic (6) [14] acids as a mixture. From EtOAc extract only $\beta$-sitosterol was obtained. The purification of $\mathrm{MeOH}$ extract afforded $\beta$-sitosteryl $\beta$-Dglucopyranoside, the flavonoids avicularin (7) $[4 a, 15]$ and
Resumen. El estudio químico de las hojas y tallos de Tibouchina urvilleana permitió aislar triterpenos de diferentes tipos: cuatro oleananos, cuatro ursanos, un glutinano y un taraxerano. También se obtuvieron dos flavonoides y dos esteroles. Adicionalmente, se realizaron pruebas de toxicidad y de actividad tópica anti-inflamatoria de los extractos. Palabras Clave: Tibouchina urvilleana, Melastomataceae, triterpenos, flavonoides, esteroles.

hispidulin 7-O- $\beta$-D-glucopyranoside (8) [16], and two mixtures, one constituted of asiatic and arjunolic acids (9 and 10) $[11 \mathrm{~b}, 17]$ and the other one of quadranoside IV (11) and arjunglucoside II (12) [17b]. Inorganic compound $\mathrm{NH}_{4} \mathrm{Cl}$ [18] was also obtained.

Compounds $\beta$-sitosterol and $\beta$-sitosteryl $\beta$-D-glucopyranoside were identified by directly comparison of their NMR and physical data, including the Rf, with those of authentic samples. The structures of the triterpenes 1-6 and 9-12 and of the flavonoids $\mathbf{7}$ and $\mathbf{8}$ were elucidated by analysis of their spectroscopic features, which were identical with their data described in the literature.

Additionally, the extracts were tested on the 12 - $O$-tetradecanoylphorbol 13-acetate (TPA) model of induced ear edema in mice [19], but the anti-inflammatory answer was not significant. Also, in the in-vitro cytotoxicity assay on the human cancer cell lines U-251, PC-3, K-562, HCT-15, MCF-7, and SKLU-1 [20], the extracts proved, unfortunately, to be moderate to poor $(63.8-1 \%)$ in the inhibition of the cancer cells growth. Nevertheless, diverse biological activities have been reported for some of the metabolites obtained in the present work. Thus, anti-inflammatory, antioxidant, antiglicative, and antibacterial activities have been reported for ursolic and
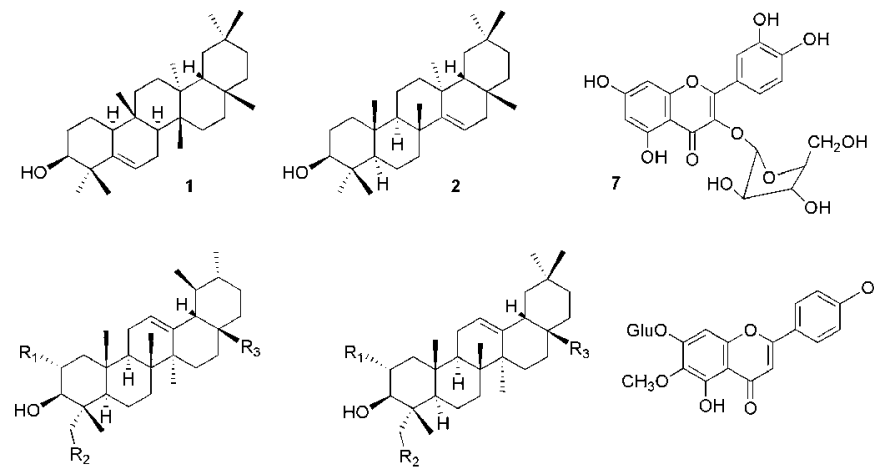

$\begin{array}{lll}3 & \mathrm{R}_{1}=\mathrm{H} & \mathrm{R}_{2}=\mathrm{H} \quad \mathrm{R}_{3}=\mathrm{CH}_{3}\end{array}$ $\begin{array}{lllll}5 & \mathrm{R}_{1}=\mathrm{H} & \mathrm{R}_{2}=\mathrm{H} & \mathrm{R}_{3}=\mathrm{CO}_{2} \mathrm{H}\end{array}$ $11 \mathrm{R}_{1}=\mathrm{OH} \mathrm{R} \mathrm{R}_{2}=\mathrm{OH} \mathrm{R} \mathrm{R}_{3}=\mathrm{CO}_{2} \mathrm{Glu}$
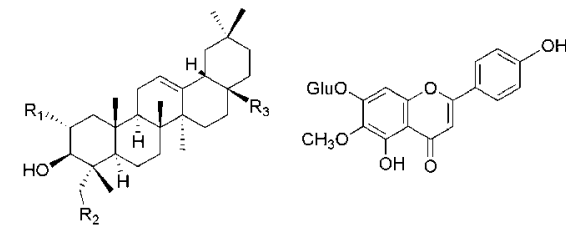

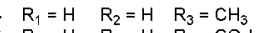

$6 \mathrm{R}_{1}=\mathrm{H} \quad \mathrm{R}_{2}=\mathrm{H} \quad \mathrm{R}_{3}=\mathrm{CO}_{2} \mathrm{H}$ $12 \mathrm{R}_{1}=\mathrm{OH} \mathrm{R}=\mathrm{OH} \mathrm{R}=\mathrm{CO}_{2} \mathrm{Glu}$ 
oleanolic acids $(\mathbf{5}, \mathbf{6})$ [21]. Antioxidant, antifungal, antibacterial, anticholinesterase, antitumoral, and antiasthmatic activities as well as beneficial therapeutics in the treatment of diabetes and the capacity of inhibition of the insect growth have been described for the arjunolic acid (10) [22]. In the case of asiatic acid (9), dermatological activities related to its ability to stimulate collagen synthesis and its anti-inflammatory activity were reported [23]. For taraxerol (2), its antidiabetic potential activity was published [24]. $\beta$-amyrin (4) was reported as a candidate for alleviating oxalate toxicity, an important urinary stone-forming constituent [25]. $\alpha$ - and $\beta$-amiryns $(\mathbf{3}, \mathbf{4})$ showed to have a potent irritant potential on mouse skin [26]. For glutinol (1), its cytotoxicity against four human cancer cell lines and its moderate anti-inflammatory activity have been reported [27]. For the flavonoid avicularin (7), its inhibitory activity of rat aldose reductase and cytotoxicity against Ehrlich ascitis carcinoma cells were described [28].

In conclusion, the chemical study of the leaves and stems of $T$. urvilleana afforded ten triterpenes (1-6, 9-12), two flavonoids $(7,8)$, two sterols, and $\mathrm{NH}_{4} \mathrm{Cl}$. Moreover, triterpenic compounds are described for the first time in the genus $\mathrm{Ti}$ bouchina.

\section{General Experimental Procedures}

Melting points were determined on a Fisher-Johns melting points apparatus and are uncorrected. IR spectra were recorded on a Bruker Tensor 27 or on a Perkin Elmer 400 spectrophotometer. NMR spectra were obtained on an Eclipse Jeol 300 MHz, a Varian-Unity $300 \mathrm{MHz}$, a Bruker Avance $300 \mathrm{MHz}$, a Varian Inova $500 \mathrm{MHz}$, or a Bruker Avance III $400 \mathrm{MHz}$ spectrometer with TMS as internal standard. EIMS $(70 \mathrm{eV})$ spectra were obtained on a Jeol JMS-AX505HA mass spectrometer and ESIMS spectra were performed in positive mode on an ESI Ion Trap Bruker Esquire 600. Vacuum column chromatography (VCC) was carried out with silica gel G 60 (Merck, Darmstadt, Germany). Flash column chromatography (FCC) was performed with silica gel 60 (230-400 mesh, Macherey-Nagel). Preparative TLC was carried out on precoated Sil G- $100 \mathrm{UV}_{254}$ plates (Macherey-Nagel). All solvents were routinely distilled prior to use.

\section{Plant Material}

Leaves and stems of Tibouchina urvilleana (DC.) Cogn. were collected in Puebla, State of Puebla, Mexico, in July 2009. A voucher specimen (MEXU 174618) was deposited at the Herbarium Nacional, Instituto de Biología, UNAM.

\section{Extraction and Isolation}

Air-dried stems and leaves were ground in a Laboratory mill (Model 4, Thomas Scientific, USA). After, the material (679 g) was put in a glass column $(10 \times 60 \mathrm{~cm})$, and with assistance of vacuum it was extracted successively with hexane, EtOAc, and $\mathrm{MeOH}$. The evaporation of solvents under reduced pressure in a Rotavapor (R-114, Büchi Labortechnik AG, Switzerland) gave the respective extracts. Hexane extract $(9 \mathrm{~g})$ was purified by VCC ( $90 \mathrm{~g}$ of silica gel) using as eluent hexane-EtOAc mixtures of increasing polarity to afford four main fractions (AD). Fraction A eluted with hexane-EtOAc 97:3 presented white solids which were crystallized on $\mathrm{CHCl}_{3}-\mathrm{MeOH}$ to yield 183.4 $\mathrm{mg}$ of glutinol (1), $\mathrm{mp} 211-213^{\circ} \mathrm{C}$. [10]. Crystallization of fraction B (obtained with hexane-EtOAc 97:3) on $\mathrm{CHCl}_{3}-\mathrm{MeOH}$ produced $8.0 \mathrm{mg}$ of taraxerol (2), $\mathrm{mp} 272-275{ }^{\circ} \mathrm{C}$ ) [11]. The mother liqueurs were recrystallized on $\mathrm{MeOH}$ to afford $93.0 \mathrm{~g}$ of $\alpha$ - and $\beta$-amyrins as a mixture (3 and 4) [11b, 12]. Fraction C, eluted with hexane-EtOAc $94: 6$, produced by crystallization on EtOH $238.0 \mathrm{mg}$ of $\beta$-sitosterol, mp 138-139 ${ }^{\circ} \mathrm{C}$ (Lit. [29] $\left.\mathrm{mp} 135-136^{\circ} \mathrm{C}\right)$. Fraction D $(485.0 \mathrm{mg})$, obtained with hexaneEtOAc $80: 20$, was purified by VCC (10 g of silica gel) using as eluent hexane- $\mathrm{Me}_{2} \mathrm{CO} 85: 15$ to produce $24.5 \mathrm{mg}$ of white crystals constituted of a mixture of ursolic and oleanolic acids (5 and 6) $[13,14]$. The purification of EtOAc extract $(14.9 \mathrm{~g})$ by VCC ( $160 \mathrm{~g}$ of silica gel) eluted with a hexane- $\mathrm{Me}_{2} \mathrm{CO}$ polarity gradient only afforded $\beta$-sitosterol $(22.4 \mathrm{mg})$. $\mathrm{MeOH}$ extract $(68 \mathrm{~g})$ was submitted to a VCC (600 $\mathrm{g}$ of silica gel) using as eluent $\mathrm{CH}_{2} \mathrm{Cl}_{2}-\mathrm{MeOH}$ mixtures of increasing polarity to yield five fractions (E-I). Fraction $\mathrm{F}\left(1.68 \mathrm{~g}\right.$, obtained with $\mathrm{CH}_{2} \mathrm{Cl}_{2}$ $\mathrm{MeOH}$ 93:7) was purified by two consecutive VCC eluted the first one with $\mathrm{CH}_{2} \mathrm{Cl}_{2}-\mathrm{MeOH}$ 95:5 and the second one with $\mathrm{CH}_{2} \mathrm{Cl}_{2}-\mathrm{Me}_{2} \mathrm{CO} 75: 25$ to produce $7.9 \mathrm{mg}$ of asiatic and arjunolic acids ( 9 and 10) as a mixture [11b,17], and $52.1 \mathrm{mg}$ of $\beta$-sitosteryl $\beta$-D-glucopyranoside, $\mathrm{mp} 290-295^{\circ} \mathrm{C}$ (desc.) (Lit. [29] mp 290-294 $\left.{ }^{\circ} \mathrm{C}\right)$. Fraction $\mathrm{G}(2.5 \mathrm{~g})$ eluted with $\mathrm{CH}_{2} \mathrm{Cl}_{2}-\mathrm{MeOH}$ 93:7 was submitted to a VCC (27 $\mathrm{g}$ of silica gel) using as eluent $\mathrm{CH}_{2} \mathrm{Cl}_{2}-\mathrm{MeOH}$ mixtures of increasing polarity to yield 81.1 $\mathrm{mg}$ of avicularin (7) [4a, 15] from $\mathrm{CH}_{2} \mathrm{Cl}_{2}-\mathrm{MeOH}$ 93:7 eluates as yellow crystals of mp $206-209^{\circ} \mathrm{C}(\mathrm{MeOH})$. Fraction $\mathrm{H}(5.6$ g, obtained with $\mathrm{CH}_{2} \mathrm{Cl}_{2}-\mathrm{MeOH}$ 90:10) was subfractioned into $\mathrm{H} 1-\mathrm{H} 3$ by a $\mathrm{VCC}$ ( $60 \mathrm{~g}$ of silica gel) eluted with $\mathrm{CH}_{2} \mathrm{Cl}_{2}-\mathrm{MeOH}$ 90:10. Subfraction H1 was submitted to a Sephadex LH-20 column using as eluent $\mathrm{MeOH}$ to afford $17.0 \mathrm{mg}$ of hispidulin 7-O- $\beta$-D-glucopyranoside (8) as yellow crystals, mp 257-259 ${ }^{\circ} \mathrm{C}$ [16], and $61.0 \mathrm{mg}$ of 7 . Subfraction $\mathrm{H} 2$ produced $57.9 \mathrm{mg}$ of 8 after a Sephadex LH-20 column eluted with MeOH. $5.1 \mathrm{mg}$ of a mixture of quadranoside IV and arjunglucoside II (11 and 12) $[17 \mathrm{~b}]$ was obtained after the purification of subfraction $\mathrm{H} 3$ by a Sephadex LH-20 column (MeOH) followed by a preparative TLC $\left(\mathrm{CH}_{2} \mathrm{Cl}_{2}-i \mathrm{PrOH} 4: 1,4 \times\right)$ and a FCC $\left(\mathrm{CH}_{2} \mathrm{Cl}_{2}-i \mathrm{PrOH}\right.$ 77:23). Fraction I (34.7 g), obtained with $\mathrm{CH}_{2} \mathrm{Cl}_{2}-\mathrm{MeOH} 85: 15$, was submitted to a VCC (350 g of silica gel) using as eluent $\mathrm{CH}_{2} \mathrm{Cl}_{2}-\mathrm{MeOH}$ mixtures of increasing polarity. From eluates of $\mathrm{CH}_{2} \mathrm{Cl}_{2}-\mathrm{MeOH} 85: 15$ were obtained $559.5 \mathrm{mg}$ of $\mathrm{NH}_{4} \mathrm{Cl}$ as white crystals (sublimate at $315^{\circ} \mathrm{C}$ ) [18]. The mother liqueurs $(250 \mathrm{mg}$ ) were purified by a VCC (3 $\mathrm{g}$ de silica gel) eluted with a $\mathrm{CH}_{2} \mathrm{Cl}_{2}-\mathrm{MeOH}$ polarity gradient to produce $89.9 \mathrm{mg}$ of $\mathrm{NH}_{4} \mathrm{Cl}$ from $\mathrm{CH}_{2} \mathrm{Cl}_{2}-\mathrm{MeOH}$ 75:25 eluates.

Glutinol (1). White crystals: $\mathrm{mp} 211-213{ }^{\circ} \mathrm{C}$ (Lit. [10] $\mathrm{mp}$ 210-213 $\left.{ }^{\circ} \mathrm{C}, 210-212{ }^{\circ} \mathrm{C}\right) ;[\alpha]_{\mathrm{D}}{ }^{25}+57.6\left(\mathrm{c} 0.25, \mathrm{CHCl}_{3}\right)$; IR (KBr) $v_{\max } 3441,2925,2866,1454,1383,1034 \mathrm{~cm}^{-1} ;{ }^{1} \mathrm{H}$ NMR 
$\left(\mathrm{CDCl}_{3}, 300 \mathrm{MHz}\right) \delta 5.63(1 \mathrm{H}$, br d, $J=6.0 \mathrm{~Hz}, \mathrm{H}-6), 3.47(1 \mathrm{H}$, dd, $J=3.3,2.4 \mathrm{~Hz}, \mathrm{H}-3), 1.16$ (3H, s, H-28), 1.14 (3H, s, H-23), 1.09 (3H, s H-26), 1.04 (3H, s, H-24), 1.00 (3H, s, H-27), 0.99 $(3 \mathrm{H}, \mathrm{s}, \mathrm{H}-30), 0.95$ (3H, s, H-29), 0.85 (3H, s, H-25); ${ }^{13} \mathrm{C}$ NMR $\left(\mathrm{CDCl}_{3}, 75 \mathrm{MHz}\right) \delta 141.6(\mathrm{C}-5), 122.1$ (C-6), 76.3 (C-3), 49.7 (C-10), 47.4 (C-18), 43.0 (C-8), 40.8 (C-14), 39.3 (C-4), 38.9 (C-22), 37.8 (C-13), 36.0 (C-16), 35.1 (C-19), 34.8 (C-9), 34.6 (C-11), 34.5 (C-30), 33.1 (C-21), 32.4 (C-28), 32.1 (C-15), 32.0 (C-29), 30.3 (C-12), 30.1 (C-17), 28.9 (C-23), 28.2 (C-20), 27.8 (C-7), 25.5 (C-24), 23.6 (C-1), 19.6 (C-27), 18.4 (C-26), 18.2 (C-2), 16.2 (C-25); EIMS m/z (rel. int.): $426[\mathrm{M}]^{+}$(7), 411 (4), 408 (3), 393 (3), 274 (100), 259 (80), 205 (30), 134 (36), 109 (29), 95 (37), 71 (24), 69 (26), 55 (21).

Taraxerol (2). White crystals: $\mathrm{mp} 272-275{ }^{\circ} \mathrm{C}$ (Lit. [11a] mp $\left.278-279{ }^{\circ} \mathrm{C}\right) ;[\alpha]_{\mathrm{D}}{ }^{25}+0.18\left(c 0.11, \mathrm{CHCl}_{3}\right) ; \mathrm{IR}\left(\mathrm{CHCl}_{3}\right) v_{\max }$ 3400, 2929, 2857, 1457, $1377 \mathrm{~cm}^{-1} ;{ }^{1} \mathrm{H}$ NMR $\left(\mathrm{CDCl}_{3}, 300\right.$ MHz) $\delta 5.53(1 \mathrm{H}, \mathrm{dd}, J=8.1,3.3 \mathrm{~Hz}, \mathrm{H}-15), 3.20(1 \mathrm{H}, \mathrm{dd}, J=$ 10.5, $4.8 \mathrm{~Hz}, \mathrm{H}-3), 2.03$ (1H, dt, $J=12.0,3.0 \mathrm{~Hz}, \mathrm{H}-7 \mathrm{a}), 1.92$ $(1 \mathrm{H}, \mathrm{dd}, J=14.7,3.0 \mathrm{~Hz}, \mathrm{H}-16 \mathrm{a}), 1.09$ (3H, s, H-26), 0.98 (3H, s, H-23), 0.95 (3H, s, H-29), 0.93 (3H, s, H-25), $0.91(6 \mathrm{H}, \mathrm{s}$, $\mathrm{H}-27, \mathrm{H}-30), 0.82(3 \mathrm{H}, \mathrm{s}, \mathrm{H}-28), 0.80$ (3H, s, H-24); ${ }^{13} \mathrm{C}$ NMR $\left(\mathrm{CDCl}_{3}, 75 \mathrm{MHz}\right) \delta 158.1$ (C-14), 116.9 (C-15), 79.1 (C-3), 55.5 (C-5), 49.3 (C-18), 48.8 (C-9), 41.3 (C-19), 39.0 (C-4), 38.8 (C-8), 38.0 (C-17), 37.74 (C-1), 37.72 (C-13), 37.6 (C-10), 36.7 (C-16), 35.1 (C-7, C-12), 33.7 (C-21), 33.4 (C-29), 33.1 (C-22), 29.9 (C-28), 29.8 (C-26), 28.8 (C-20), 28.0 (C-23), 27.2 (C-2), 25.9 (C-27), 21.3 (C-30), 18.8 (C-6), 17.5 (C-11), 15.5 (C-24, C-25); EIMS $m / z$ (rel. int.): $426\left[\right.$ [M] $^{+}$(27), 411 (24), 302 (57), 287 (37), 218 (29), 204 (100), 189 (19), 135 (31), 95 (19), 69 (27), 55 (28).

$\boldsymbol{\alpha}$ - and $\boldsymbol{\beta}$ - Amyrins (3, 4). IR (KBr) $v_{\max } 3285,2918,2850$, 1460, 1381, 1362, 1033, $995 \mathrm{~cm}^{-1}$; compound 3: ${ }^{1} \mathrm{H}$ NMR $\left(\mathrm{CDCl}_{3}, 300 \mathrm{MHz}\right) \delta 5.13(1 \mathrm{H}, \mathrm{dd}, J=3.6,3.3 \mathrm{~Hz}, \mathrm{H}-12), 3.23$ $(1 \mathrm{H}, \mathrm{dd}, J=10.5,5.4 \mathrm{~Hz}, \mathrm{H}-3), 1.07$ (3H, s, H-27), $1.01(3 \mathrm{H}$, s, H-26), 1.00 (3H, s, H-23), 0.96 (3H, s, H-25), 0.92 (3H, d, $J$ $=7.5 \mathrm{~Hz}, \mathrm{H}-30), 0.80(3 \mathrm{H}, \mathrm{s}, \mathrm{H}-28), 0.79(3 \mathrm{H}, \mathrm{d}, J=6.0 \mathrm{~Hz}$, $\mathrm{H}-29), 0.79(3 \mathrm{H}, \mathrm{s}, \mathrm{H}-24) ;{ }^{13} \mathrm{C} \mathrm{NMR}\left(\mathrm{CDCl}_{3}, 75 \mathrm{MHz}\right) \delta 139.6$ (C-13), 124.4 (C-12), 79.0 (C-3), 59.0 (C-18), 55.1 (C-5), 47.7 (C-9), 42.0 (C-14), 41.5 (C-22), 39.6 (C-19, C-20), 38.8 (C-1, C-4, C-8), 36.9 (C-10), 33.7 (C-17), 32.9 (C-7), 31.2 (C-21), 29.7 (C-16), 28.7 (C-28), 28.1 (C-23), 27.2 (C-2), 26.6 (C-15), 23.3 (C-11, C-27), 21.4 (C-30), 18.4 (C-6), 17.5 (C-29), 16.8 (C-26), 15.6 (C-24, C-25); compound 4: ${ }^{1} \mathrm{H}$ NMR $\left(\mathrm{CDCl}_{3}, 300\right.$ $\mathrm{MHz}) \delta 5.18(1 \mathrm{H}, \mathrm{dd}, J=3.6,3.3 \mathrm{~Hz}, \mathrm{H}-12), 3.24(1 \mathrm{H}, \mathrm{dd}, J$ $=10.2,5.1 \mathrm{~Hz}, \mathrm{H}-3), 1.13(3 \mathrm{H}, \mathrm{s}, \mathrm{H}-27), 1.00(3 \mathrm{H}, \mathrm{s}, \mathrm{H}-26)$, 0.97 (3H, s, H-28), 0.94 (3H, s, H-25), 0.87 (6H, s, H-29, H30), 0.83 (3H, s, H-23), 0.79 (3H, s, H-24); ${ }^{13} \mathrm{C} \mathrm{NMR}\left(\mathrm{CDCl}_{3}\right.$, $75 \mathrm{MHz}) \delta 145.2$ (C-13), 121.7 (C-12), 79.0 (C-3), 55.1 (C-5), 47.6 (C-9), 47.2 (C-18), 46.8 (C-19), 41.7 (C-14), 39.8 (C-8), 38.8 (C-4), 38.6 (C-1), 37.4 (C-22), 36.9 (C-10), 34.7 (C-21), 33.3 (C-29), 32.6 (C-7), 31.1 (C-20), 28.4 (C-28), 28.1 (C-15, C-23), 27.0 (C-27), 26.1 (C-2, C-16), 23.7 (C-30), 23.5 (C-11), 18.4 (C-6), 16.8 (C-26), 15.7 (C-25), 15.5 (C-24).

Ursolic (5) and oleanolic (6) acids. IR (KBr) $v_{\max } 3412,2925$, 2858, 1688, 1455, 1382, 1030, $996 \mathrm{~cm}^{-1}$; compound 5: ${ }^{1} \mathrm{H}$ NMR $\left(\mathrm{CDCl}_{3}+\mathrm{DMSO}-d_{6}, 300 \mathrm{MHz}\right) \delta 5.24(1 \mathrm{H}, \mathrm{t}, J=3.6 \mathrm{~Hz}, \mathrm{H}-11)$,
$3.18(1 \mathrm{H}, \mathrm{dd}, J=8.3,6.4 \mathrm{~Hz}, \mathrm{H}-3), 2.20(1 \mathrm{H}, \mathrm{brd}, J=11.1 \mathrm{~Hz}$, $\mathrm{H}-18), 1.14$ (3H, s, H-27), 1.08 (6H, s, H-23, H-26), 0.99 (3H, s, H-24), $0.93(3 \mathrm{H}, \mathrm{d}, J=7.5 \mathrm{~Hz}, \mathrm{H}-29), 0.85(3 \mathrm{H}, \mathrm{d}, J=6.3$ $\mathrm{Hz}, \mathrm{H}-30), 0.78$ (3H, s, H-25); ${ }^{13} \mathrm{C}$ NMR $\left(\mathrm{CDCl}_{3}+\mathrm{DMSO}-d_{6}\right.$, $75 \mathrm{MHz}) \delta 179.6$ (C-28), 138.0 (C-13), 125.0 (C-12), 78.3 (C-3), 55.0 (C-5), 52.5 (C-18), 47.3 (C-9, C-17), 41.8 (C-14), 40.1 (C-8), 39.3 (C-4), 38.8 (C-19), 38.6 (C-20), 38.5 (C-10), 38.4 (C-1), 36.5 (C-22), 32.7 (C-7), 32.2 (C-21), 30.4 (C-15), 27.9 (C-23), 27.0 (C-2), 23.9 (C-16), 23.2 (C-27), 23.0 (C-11), 20.9 (C-30), 18.1 (C-6), 16.8 (C-26, C-29), 16.7 (C-24, C-25); compound 6: ${ }^{1} \mathrm{H}$ NMR $\left(\mathrm{CDCl}_{3}+\mathrm{DMSO}-d_{6}, 300 \mathrm{MHz}\right) \delta 5.27$ $(1 \mathrm{H}, \mathrm{t}, J=3.6 \mathrm{~Hz}, \mathrm{H}-11), 3.18(1 \mathrm{H}, \mathrm{dd}, J=9.0,5.7 \mathrm{~Hz}, \mathrm{H}-3)$, $2.84(1 \mathrm{H}, \mathrm{dd}, J=14.4,4.5 \mathrm{~Hz}, \mathrm{H}-18), 1.14$ (3H, s, H-27), 1.08 $(3 \mathrm{H}, \mathrm{s}, \mathrm{H}-23), 0.99$ (3H, s, H-24), 0.92 (3H, s, H-30), 0.91 (3H, s, H-29), 0.89 (3H, s, H-25), 0.80 (3H, s, H-26); ${ }^{13} \mathrm{C}$ NMR $\left(\mathrm{CDCl}_{3}+\right.$ DMSO- $\left.d_{6}, 75 \mathrm{MHz}\right) \delta 179.8(\mathrm{C}-28), 143.8(\mathrm{C}-13)$, 121.8 (C-12), 78.3 (C-3), 55.0 (C-5), 47.3 (C-9), 45.8 (C-17, C-19), 40.9 (C-18), 40.6 (C-14), 40.4 (C-8), 40.1 (C-4), 38.2 (C-1), 36.7 (C-10), 33.7 (C-7), 32.7 (C-29), 32.5 (C-21, C-22), 29.3 (C-20), 27.9 (C-23), 27.8 (C-15), 27.4 (C-2), 25.6 (C-27), 23.1 (C-16), 22.8 (C-11), 23.3 (C-30), 18.1 (C-6), 16.7 (C-26), 15.5 (C-24, C-25).

Avicularin (7). Yellow crystals: $\mathrm{mp}$ 206-209 ${ }^{\circ} \mathrm{C}$ (Lit. [4b, 15b] mp 170-175 $\left.{ }^{\circ} \mathrm{C}, \mathrm{mp} 216-217^{\circ} \mathrm{C}\right) ;[\alpha]_{\mathrm{D}}^{25}-184.5$ (c 0.35, $\mathrm{MeOH}$ ); IR (Nujol) $v_{\max } 3284,1654,1605,1559,1364,1270$, $1199,1114 \mathrm{~cm}^{-1} ;{ }^{1} \mathrm{H}$ NMR (MeOH- $\left.d_{4}, 300 \mathrm{MHz}\right) \delta 7.52(1 \mathrm{H}$, d, $J=2.1 \mathrm{~Hz}, \mathrm{H}-2$ '), $7.48(1 \mathrm{H}, \mathrm{dd}, J=8.4,2.1 \mathrm{~Hz}, \mathrm{H}-6$ '), 6.89 $(1 \mathrm{H}, \mathrm{d}, J=8.4 \mathrm{~Hz}, \mathrm{H}-5$ '), $6.38(1 \mathrm{H}, \mathrm{d}, J=2.1 \mathrm{~Hz}, \mathrm{H}-8), 6.15$ $(1 \mathrm{H}, \mathrm{d}, J=2.1 \mathrm{~Hz}, \mathrm{H}-6), 5.46$ (1H, d, $J=1.0 \mathrm{~Hz}, \mathrm{H}-1$ ' '), 4.32 $(1 \mathrm{H}, \mathrm{dd}, J=3.0,1.0 \mathrm{~Hz}, \mathrm{H}-2$ ') $) 3.91(1 \mathrm{H}, \mathrm{dd}, J=5.1,3.0 \mathrm{~Hz}$, H-3'), 3.87 (1H, ddd, $J=6.0,5.1,3.9 \mathrm{~Hz}, \mathrm{H}-4$ '”), $3.5(2 \mathrm{H}, \mathrm{m}$, H-5' '); ${ }^{13} \mathrm{C}$ NMR (MeOH- $\left.d_{4}, 75 \mathrm{MHz}\right) \delta 180.0$ (C-4), 166.1 (C7), 163.1 (C-5), 159.3 (C-2), 158.6 (C-9), 149.8 (C-4'), 146.4 (C-3'), 134.9 (C-3), 123.1 (C-1'), 123.0 (C-6'), 116.9 (C-2'), 116.5 (C-5'), 109.6 (C-1'), 105.6 (C-10), 99.9 (C-6), 94.8 (C8), 88.1 (C-4"), 83.3 (C-2'”), 78.7 (C-3"), 62.6 (C-5'); ESIMS $\mathrm{m} / \mathrm{z} 457[\mathrm{M}+\mathrm{Na}]^{+}$.

Hispidulin 7- $\boldsymbol{O}-\boldsymbol{\beta}$-D-glucopyranoside (8). Yellow crystals: mp 257-259 ${ }^{\circ} \mathrm{C}$ (Lit. [16a] mp 256-258 $\left.{ }^{\circ} \mathrm{C}\right) ;[\alpha]_{\mathrm{D}}{ }^{25}-45.5(c$ $0.24, \mathrm{MeOH}$ ); IR (Nujol) $v_{\max } 3308,1644,1597,1564,1460$, $1376,1357,1249,1102 \mathrm{~cm}^{-1} ;{ }^{1} \mathrm{H}$ NMR (DMSO- $d_{6}, 500 \mathrm{MHz}$ ) $\delta 7.94$ (2H, d, $J=9.0 \mathrm{~Hz}, \mathrm{H}-2^{\prime}$ ', H-6'), 7.01 (1H, s, H-8), 6.93 $\left(2 \mathrm{H}, \mathrm{d}, J=9.0 \mathrm{~Hz}, \mathrm{H}-3^{\prime}, \mathrm{H}-5^{\prime}\right), 6.84(1 \mathrm{H}, \mathrm{s}, \mathrm{H}-3), 5.10(1 \mathrm{H}, \mathrm{d}$, $J=7.0 \mathrm{~Hz}, \mathrm{H}-1$ ' '), $3.76\left(3 \mathrm{H}, \mathrm{s}, \mathrm{OCH}_{3}\right), 3.74(1 \mathrm{H}, \mathrm{m}, \mathrm{H}-6 \mathrm{a}$ ' '), 3.48 (1H, m, H-6b'), 3.45 (1H, m, H-4'), 3.33 (1H, m, H-3'), 3.31 (1H, m, H-2"'), 3.20 (1H, dd, $J=9.0,8.0 \mathrm{~Hz}, \mathrm{H}-5$ "'); ${ }^{13} \mathrm{C}$ NMR (DMSO- $\left.d_{6}, 125 \mathrm{MHz}\right) \delta 182.3$ (C-4), 164.3 (C-2), 161.3 (C-4'), 156.5 (C-7), 152.4 (C-9), 152.1 (C-5), 132.5 (C-6), 128.6 (C-2', C-6'), 121.1 (C-1'), 116.0 (C-3', C-5'), 105.7 (C10), 102.7 (C-3), 100.2 (C-1'), 94.4 (C-8), 77.3 (C-4'), 76.7 (C-2'), 73.2 (C-3'), 69.6 (C-5'), 60.6 (C-6”), $60.3\left(\mathrm{OCH}_{3}\right)$; ESIMS $m / z 485[\mathrm{M}+\mathrm{Na}]^{+}$.

Asiatic (9) and arjunolic (10) acids. IR (KBr) $v_{\max } 3319$, 2921, 2853, 1690,1457, 1382, $1035 \mathrm{~cm}^{-1}$; compound 9: ${ }^{1} \mathrm{H}$ NMR (pyridine- $\left.d_{5}, 300 \mathrm{MHz}\right) \delta 5.49(1 \mathrm{H}, \mathrm{t}, J=3.6 \mathrm{~Hz}, \mathrm{H}-$ 12), 4.3-4.2 (4H, m, H-2, H-3, H-23), $2.63(1 \mathrm{H}, \mathrm{d}, J=9.6$ 
$\mathrm{Hz}, \mathrm{H}-18), 1.22$ (3H, s, H-26), 1.09 (6H, s, H-25, H-27), 1.07 $(3 \mathrm{H}, \mathrm{s}, \mathrm{H}-24), 0.98(3 \mathrm{H}, \mathrm{d}, J=7.0 \mathrm{~Hz}, \mathrm{H}-29), 0.93(3 \mathrm{H}, \mathrm{d}, J$ $=7.0 \mathrm{~Hz}, \mathrm{H}-30) ;{ }^{13} \mathrm{C}$ NMR (pyridine- $\left.d_{5}, 75 \mathrm{MHz}\right) \delta 180.2(\mathrm{C}-$ 28), 139.3 (C-13), 125.6 (C-12), 78.3 (C-3), 68.9 (C-2), 66.6 (C-23), 53.6 (C-18), 48.0 (C-5, C-17), 47.9 (C-9), 47.8 (C-1), 43.7 (C-4), 42.2 (C-14), 40.1 (C-8), 39.5 (C-19), 39.4 (C-20), 38.3 (C-10), 37.5 (C-22), 32.9 (C-7), 31.1 (C-21), 28.7 (C-15), 24.9 (C-16), 23.9 (C-27), 23.7 (C-11), 21.4 (C-30), 18.6 (C-6), 17.6 (C-26), 17.4 (C-25, C-29), 14.4 (C-24); compound 10: ${ }^{1} \mathrm{H}$ NMR (pyridine- $\left.d_{5}, 300 \mathrm{MHz}\right) \delta 5.49(1 \mathrm{H}, \mathrm{t}, J=3.6 \mathrm{~Hz}, \mathrm{H}-12)$, 4.3-4.2 (4H, m, H-2, H-3, H-23), $3.30(1 \mathrm{H}, \mathrm{dd}, J=13.6,4.4$ $\mathrm{Hz}, \mathrm{H}-18), 1.22$ (3H, s, H-26), $1.16(3 \mathrm{H}, \mathrm{s}, \mathrm{H}-27), 1.09$ (3H, $\mathrm{s}, \mathrm{H}-25), 1.07(3 \mathrm{H}, \mathrm{s}, \mathrm{H}-24), 1.01(3 \mathrm{H}, \mathrm{s}, \mathrm{H}-30), 0.93(3 \mathrm{H}, \mathrm{s}$, $\mathrm{H}-29$ ); ${ }^{13} \mathrm{C}$ NMR (pyridine- $\left.d_{5}, 75 \mathrm{MHz}\right) \delta 179.9$ (C-28), 144.9 (C-13), 125.5 (C-12), 78.3 (C-3), 68.9 (C-2), 66.6 (C-23), 48.1 (C-9), 47.9 (C-5), 47.8 (C-1), 46.7 (C-17), 46.4 (C-19), 42.0 (C-18), 43.7 (C-4), 42.2 (C-14), 39.9 (C-8), 38.5 (C-10), 34.2 (C-21), 33.2 (C-22, C-29), 32.9 (C-7), 31.0 (C-20), 28.3 (C-15), 26.2 (C-27), 24.0 (C-16), 23.8 (C-30), 23.7 (C-11), 18.6 (C-6), 17.6 (C-25), 17.5 (C-26), 14.4 (C-24).

Quadranoside IV (11) and arjunglucoside II (12). IR (Nujol) $v_{\max } 3369,1732,1637 \mathrm{~cm}^{-1}$; compound 11: ${ }^{1} \mathrm{H}$ NMR (pyridine$\left.d_{5}, 500 \mathrm{MHz}\right) \delta 6.25(1 \mathrm{H}, \mathrm{d}, J=8.5 \mathrm{~Hz}, \mathrm{H}-1$ ' $), 5.41(1 \mathrm{H}, \mathrm{brs}$, H-12), 4.3-4.23 (2H, m, H-2, H-3), 4.45-4.19 (5H, m, H-2', H-3', H-4', H-6', H-23a), 4.03 (1H, m, H-5'), 3.70 (1H, d, $J=$ $10.0 \mathrm{~Hz}, \mathrm{H}-23 \mathrm{~b}), 2.50(1 \mathrm{H}$, brd, $J=11.0 \mathrm{~Hz}, \mathrm{H}-18), 1.18(3 \mathrm{H}$, s, H-26), 1.10 (3H, s, H-25), 1.09 (3H, s, H-27), 1.06 (3H, s, $\mathrm{H}-24), 0.90(3 \mathrm{H}, \mathrm{d}, J=6.0 \mathrm{~Hz}, \mathrm{H}-29), 0.86(3 \mathrm{H}, \mathrm{s}, \mathrm{H}-30) ;{ }^{13} \mathrm{C}$ NMR (pyridine- $\left.d_{5}, 125 \mathrm{MHz}\right) \delta 181.3(\mathrm{C}-28), 137.0(\mathrm{C}-13)$, 124.0 (C-12), 95.7 (C-1'), 79.2 (C-5'), 78.9 (C-3'), 74.0 (C2'), 71.2 (C-3, C-4'), 68.9 (C-2), 66.5 (C-23), 62.2 (C-6'), 53.3 (C-18), 48.2 (C-17), 48.0 (C-5), 47.9 (C-9), 47.8 (C-1), 43.6 (C-4), 42.2 (C-14), 40.2 (C-8), 39.2 (C-19), 39.1 (C-20), 38.3 (C-10), 36.8 (C-21, C-22), 32.8 (C-7), 28.6 (C-15), 24.6 (C-16), 23.7 (C-11), 23.6 (C-27), 21.2 (C-30), 18.5 (C-6), 17.6 (C-26), 17.4 (C-25), 17.3 (C-29), 14.4 (C-24); compound 12: ${ }^{1} \mathrm{H}$ NMR (pyridine- $\left.d_{5}, 500 \mathrm{MHz}\right) \delta 6.32(1 \mathrm{H}, \mathrm{d}, J=8.0 \mathrm{~Hz}, \mathrm{H}-1$ '), 5.43 (1H, brs, H-12), 4.3-4.23 (2H, m, H-2, H-3), 4.45-4.19 (5H, m, H-2', H-3', H-4', H-6', H-23a), 4.03 (1H, m, H-5'), 3.70 $(1 \mathrm{H}, \mathrm{d}, J=10.0 \mathrm{~Hz}, \mathrm{H}-23 \mathrm{~b}), 3.17(1 \mathrm{H}, \mathrm{dd}, J=14.0,4.0 \mathrm{~Hz}$, H-18), 1.15 (6H, s, H-26, H-27), 1.09 (3H, s, H-25), 1.06 (3H, s, H-24), 0.86 (6H, s, H-29, H-30); ${ }^{13} \mathrm{C}$ NMR (pyridine- $d_{5}, 125$ MHz) $\delta 181.3$ (C-28), 143.5 (C-13), 124.0 (C-12), 95.7 (C-1'), 79.3 (C-5'), 78.9 (C-3'), 74.1 (C-2'), 71.2 (C-3), 71.1 (C-4'), 68.9 (C-2), 66.5 (C-23), 62.3 (C-6'), 48.1 (C-5), 47.9 (C-1), 47.8 (C-9), 47.0 (C-17), 46.1 (C-19), 43.6 (C-4), 41.7 (C-14, C-18), 40.0 (C-8), 38.4 (C-10), 33.9 (C-21), 33.2 (C-7), 33.0 (C-29), 32.5 (C-22), 30.7 (C-20), 28.2 (C-15), 26.0 (C-27), 23.8 (C-11), 23.7 (C-30), 23.4 (C-16), 18.4 (C-6), 17.7 (C-26), 17.6 (C-25), 14.4 (C-24).

\section{Acknowledgements}

I am indebted to Héctor Ríos, Elizabeth Huerta, Beatriz Quiroz, Isabel Chávez, Rubén Gaviño, Carmen Márquez, Javier Pérez, and Rocío Patiño from the Instituto de Química, Universidad Nacional Autónoma de México, for technical assistance.

\section{References}

1. Wu, W.; Dai, S.; Bao, H.; Zhou, R. Biochem. Syst. Ecol. 2009, 37, 640-644.

2. Machado, K. R.; Arnold, N.; Wessjohann, L. Biochem. Syst. Ecol. 2009, 37, 63-65.

3. Colorado, A.; Maya, D. C.; Díaz, G. S. J.; Isaza, M. J. H.; Tapias, I. L. J.; Veloza, L. A.; Ramírez, A. L. S. Flavonoides del extracto isopropanol-agua de Tibouchina ciliaris. Scientia et Técnica, Abril, 2007, Vol. XIII, No. 33, Universidad Tecnológica de Pereira, Pereira, Colombia, pp. 355-357.

4. a) Sirat, H. M.; Rezali, M. F.; Ujang, Z. J Agric. Food Chem. 2010, 58, 10404-10409. b) Yoshida, T.; Nakada, F.; Okuda, T. Chem Pharm. Bull. 1999, 47, 824-827. 4c) Harborne, J. B. Phytochemistry 1964, 3, 151-160.

5. Yoshida, T.; Amakura, Y.; Yokura, N.; Ito, H.; Isaza, J. H.; Ramírez, S.; Pelaez, D. P.; Renner, S. S. Phytochemistry 1999, $52,1661-1666$.

6. Motta, L. B.; Kraus, J. E.; Salatino, A.; Salatino, M. L. F. Biochem. Syst. Ecol. 2005, 33, 971-981.

7. Bobbio, F. O.; Bobbio, P. A.; Degáspari, C. H. Food Chem. 1985, 18, 153-159.

8. a) Francis, F. J.; Draetta, I.; Baldini, V.; Iaderoza, M. J. Am. Soc. Hort. Sci. 1982, 107, 789-791. b) Okumura, F.; Soares, M. H. F. B.; Cavalheiro, T. G. Quim. Nova 2002, 25, 680-683.

9. Terahara, N.; Suzuki, H.; Toki, K.; Kuwano, H.; Saito, N.; Honda, T. J. Nat. Prod. 1993, 56, 335-340.

10. a) Matsunaga, S.; Tanaka, R., Akagi, M. Phytochemistry 1988, 27, 535-537. b) El-Seedi, H. R. Nat Prod Res. 2005, 19, 197-202.

11. a) Sakurai, N.; Yaguchi, Y.; Inoue, T. Phytochemistry 1987, 26, 217-219. b) Viqar Uddin Ahmad, Atta-ur-Rahman. Handbook of Natural Products Data. Vol 2, Elsevier, Amsterdam, 1994, 21-22, 320-322, 516-517, 716-717. c) Lee, J. H.; Lee, K. T.; Yang, J. H.; Baek, N. I.; Kim, D. K. Arch. Pharm. Res. 2004, 27, 53-56.

12. a) Toriumi, Y.; Kakuda, R.; Kikuchi, M.; Yaoita, Y.; Kikuchi, M. Chem. Pharm. Bull. 2003, 51, 89-91. b) Ramasamy, D.; Saraswathy, A. Food Chem. 2014, 145, 970-975.

13. Lemes, G. F.; Ferri, P. H. Quim. Nova. 2011, 34, 39-42.

14. Hu, H. B.; Zheng, X.-D.; Jian, Y.-F.; Liu, J.-X.; Zhu, J.-H. Arch. Pharm. Res. 2011, 34, 1097-1105.

15. a) Schieber, A.; Hilt, P.; Conrad, J.; Beifuss, U.; Carle, R. J. Sep. Sci. 2002, 25, 361-364. b) Zhang, X.; Thuong, P. T.; Jin, W. Y.; Su, N. D.; Sok, D. E.; Bae, K.; Kang, S. S. Arch. Pharm. Res. 2005, 28, 22-27.

16. a) Aritomi, M. Chem. Pharm. Bull. 1963, 15, 432-434. b) Hase, T.; Ohtani, K.; Kasai, R.; Yamasaki, K.; Picheansoonthon, C. Phytochemistry 1995, 40, 287-290.

17. a) Jeong, B.-S.; Lee, M. K.; Kim, Y. C.; Lee, E.-S. Arch. Pharm. Res. 2007, 30, 282-289. b) Bisoli, E.; Silva, G. W.; Hamerski, L.; Tieppo, C.; Rodríguez, G. F. Molecules 2008, 13, 2717-2728.

18. Richard A. Nyquist, Ronald O. Kagel. Handbook of Infrared and Raman Spectra of Inorganic Compounds and Organic Salts. Vol 4, Academic Press Inc., San Diego USA, 1996, 715.

19. Arciniegas, A.; Pérez-Castorena, A. L.; Nieto-Camacho, A.; Villaseñor, J. L.; Romo de Vivar, A. J. Mex. Chem. Soc. 2009, 53, 229-232.

20. Arciniegas, A.; Polindara, L. A.; Pérez-Castorena, A. L.; García, A. M.; Ávila, G.; Villaseñor, J. L.; Romo de Vivar, A. Z. Naturforsch. 2011, 66c, 115-122.

21. a) Yin, M.-C.; Lin, M.-C.; Mong, M.-C.; Lin, C.-Y. J. Agric. Food Chem. 2012, 60, 7697-7701. b). Acebey-Castellon, I. L.; Voutquenne-Nazabadioko, L.; Mai, H. D. T.; Roseau, N.; Boutha- 
gane, N.; Muhammad, D.; Le Magrex Debar, E.; Gangloff, S. C.; Litaudon, M.; Sevenet, T.; Hung, N. V.; Lavaud, C. J. Nat. Prod. 2011, 74, 163-168.

22. Manna, P.; Sil, P. C. Free Radical Res. 2012, 46, 815-830.

23. Aguirre, M. C.; Delporte, C.; Backhouse, N.; Erazo, S.; Letelier, M. E.; Cassels, B. K.; Silva, X.; Alegría, S.; Negrete, R. Bioorg. Med. Chem. 2006, 14, 5673-5677.

24. Sangeetha, K. N.; Sujatha, S.; Muthusamy, V. S.; Anand, S.; Nithya, N.; Velmurugan, D.; Balakrishnan, A.; Lakshmi, B. S. Biochim. Biophys. Acta-Gen. Subjects. 2010, 1800, 359-366.

25. Geetha, K.; Venkappayya, D.; Manavalan, R. Asian J. Chem. 2010, 22, 6547-6552.
26. Saeed, M. A.; Sabir, A. W. J. Asian Nat. Prod. Res. 2003, 5, 3541.

27. Ding, Y.; Liang, C.; Kim, J. H.; Lee, Y.-M.; Hyun, J.-H.; Kang, H.-K.; Kim, J.-A.; Min, B. S.; Kim, Y. H. Bioorg. Med. Chem. Lett. 2010, 20, 1528-1535.

28. a) Shimizu, M.; Ito, T.; Tarashima, S.; Hayashi, T.; Arisawa, M.; Morita, N.; Kurokawa, S.; Ito, K.; Hashimoto, Y. Phytochemistry 1984, 23, 1885-1888. b) Marzouk, M. S.; Soliman, F. M.; Shehata, I. A.; Rabee, M.; Fawzy, G. A. Nat. Prod. Res. 2007, 21, 436-443. 29. Rawat, M. S. M.; Negi, D. S.; Panwar, M. S.; Pant, G. Fitoterapia 1988, 59, 248-249. 